\title{
Evaluating Accessibility in Ubiquitous Environments: a Case Study with Museum Installations
}

\author{
Josiane Rosa de Oliveira Gaia Pimenta ${ }^{1,2}$, Emanuel Felipe Duarte ${ }^{1}$, \\ Maria Cecília Calani Baranauskas ${ }^{1,3}$ \\ ${ }^{1}$ Institute of Computing, University of Campinas (UNICAMP), Campinas/SP - Brazil \\ ${ }^{2}$ Federal Institute of São Paulo (IFSP), Hortolândia/SP - Brazil \\ ${ }^{3}$ PPGInf, Federal University of Paraná (UFPR), Curitiba/PR - Brazil \\ josiane.o.gaia@gmail.com, \{emanuel.duarte, cecilia\}@ic.unicamp.br
}

\begin{abstract}
Ubiquitous environments dynamics brings new possibilities of interaction with technology and with people in the same environment. This paper investigates accessibility challenges in ubiquitous environments through existing formal principles (Universal Design principles, WCAG 2.1 and Good Practices of Urban Design). A case study at a museum scenario sheds light on the need for adaptations and lack of coverage of the principles. The main contributions of this paper are: 1) suitability analysis of existing principles and formal standards for accessibility transposed to the analyzed scenario; 2) from the analysis result, insights on missing aspects that might be brought to the design of ubiquitous computing scenarios accessible for all.
\end{abstract}

\section{Introduction}

The evolution of technology has brought new ways of interacting with computers. Ubiquitous computing, for instance, departs from personal computers as the focus of attention, instead of claiming computers should be in the periphery of the attention [Takayama 2017]. New forms of interactions made possible by ubiquitous computing through sensors and actuators spread out in the environment have been used in numerous contexts such as public spaces. However, as the interaction transcends computer direct use, being into the physical environment, unforeseen accessibility issues may arise, and current principles and guidelines may not be enough to address them. In this paper we investigate accessibility in ubiquitous environments, considering the specific scenario of a museum and three interactive installations exhibited in it.

As argued by [Emiliani and Stephanidis 2005], it is important to plan designs to attend all the possible needs of users without the need of making a posteriori adaptations to allow access. Equitable access is indeed a concern of big organizations, such as the United Nations and its sustainable 2030 agenda [United Nations 2013]. In this direction, the concern for universal access [Stephanidis 2009] and the new forms of interaction afforded by ubiquitous computing raises discussions about accessibility, such as how to allow access to everyone without discrimination in such scenarios.

The objective of this paper is to investigate the applicability of accessibility evaluation principles and formal standards to ubiquitous technology environments in a museum scenario. Two research questions are proposed: $\boldsymbol{R} Q \mathbf{1}$ : Are literature guides sufficient to 
evaluate accessibility in ubiquitous environments?; and $\boldsymbol{R Q} 2$ : What are the drawbacks to evaluate accessibility in ubiquitous environments? We raised existing accessibility principles and formal standards, and then applied the guidelines on a case study aiming at answering our two research questions.

The remaining of this paper is organized as follows: in Section 2 we present a background on ubiquitous computing and accessibility, related work and formal principles; in Section 3 we describe the museum workshop and its interactive installations as a case study of accessibility evaluation in ubiquitous environments; in Section 3.2 we present our main findings and their implications; Lastly, in Section 4 we present our conclusions and directions for future work.

\section{Background and Related Work}

Ubiquitous computing involves many aspects, from abstract concepts such as transparency, embodiment, and drawing computers out of their shells to more concrete ones such as different sizes and shapes, network connections, prices, power consumption, and displays. This kind of technology fades into the background where the user's interaction becomes unconscious and natural, providing seamless and unobtrusive interactions. This invisible technology also enhances social aspects by changing technology from a barrier to a transparent issue, fitting into the human environment [Weiser 1991].

Ubiquitous environments, such as Mood Conductor [Morgan and Gunes 2013], TangiTime [Mendoza and Baranauskas 2019] and The Magic of Science [Duarte et al. 2019] benefit museums and other scenarios by providing immersive experiences and new forms of interaction with technology and art. Mood Conductor [Morgan and Gunes 2013] is an interactive art experiment conducted during the Olympic Games in 2012. It consists of capturing emotions and movements of the participants and projecting different light styles at the rim of the London Eye. The movements are captured using a Kinect and a heart monitor. In accordance with the movement and the emotions, a different light style (Wave, Spectrum and Fire) is projected at the London Eye. TangiTime [Mendoza and Baranauskas 2019], in turn, is a tabletop tangible user interface designed to explore the concept of Deep Time and provide learning through the interactions with objects of the installation and with other people participating in the installation. Five objects (meteorite, volcano, dragonfly, tyrannosaurus rex and triceratops) react when placed on the table over the correct geological era. The table presents an animation according to the era, and the objects had lighting, vibration, sounds or even interaction among themselves. The experiment also allowed social interactions among participants. Lastly, The Magic of Science, a workshop conducted in an exploratory science museum with three different installations, the case study of this paper, is detailed in Section 3.

The new possibilities of ubiquitous environments, however, makes us wonder whether they are accessible to everyone. The World Health Organization (WHO) defines disability as the "term for impairments, activity limitations and participation restrictions. It denotes the negative aspects of the interaction between an individual (with a health condition) and that individual's contextual factors (environmental and personal factors)" [World Health Organization 2013]; around a billion people, approximately 15\% of the world's population, live with some disability [World Health Organization 2011]. 
The United Nations (UN) highlights the need of non-discrimination and equitable access which benefit all people, with or without disabilities. An inclusive society must support every individual equally, enabling their full participation in social, economic and political life [United Nations 2013].

As related works, accessibility in museums was investigated by Hayashi and Baranauskas (2017). The study mentions tactile floors and maps to allow access to visual impaired people, noting they were available only in a few museums visited in Brazil. It is argued that accessibility should be considered throughout the entire facility, and not only within exhibits. Finally, the concept of socioenactive system is discussed presenting the importance of the social side of the interaction [Hayashi and Baranauskas 2017]. Another related work, a systematic review focusing on multi-device inclusive environment was presented by [Bittencourt et al. 2015]. It highlights the need of considering accessibility as a non-functional requirement, and states that the social conditions of the users must be taken into account. The authors present the challenges of involving users with different needs in the system development cycle, the need of new software engineering approaches to support user system adaptations at design and runtime, and the necessity of methods to evaluate proposed solutions. Lastly, [Gonçalves et al. 2020] conducted an exploratory study of accessibility in pervasive and ubiquitous computing. Solutions designed for visual, hearing, motor and cognitive impairments were categorized and distributed into WCAG 2.0 principles.

\subsection{Accessibility Principles and Formal Standards}

We selected three sets of principles or formal standards: 1) the Principles of Universal Design [Connell et al. 1997] cover three different perspectives: physical, technological and, reach [Connell et al. 1997]; 2) the Web Content Accessibility Guidelines (WCAG) [W3C 2018] support web accessibility with the current stable version being 2.1 (further versions 2.2 and 3.0 are under development, and might cover wearable and other web of things devices ); and 3) the Good Practices of Accessible Urban Development [United Nations 2015] focused on physical environments.

A summary of the Universal Design Principles and the WCAG are presented in Tables 2, and 1, respectively. And the principles of the Good Practices of Accessible Urban Development are: 1) Promoting accessibility as a collective good and a key component in urban policy, design, planning and development; 2) Accessible housing and built infrastructures as key elements for sustainable and inclusive cities; 3) Accessible transportation, public spaces and public services; 4) Accessible information and commu-

Table 1. Principles of the WCAG 2.1 [W3C 2018].

\begin{tabular}{ll}
\hline Principle & Description \\
\hline 1) Perceivable & $\begin{array}{l}\text { Information and user interface components must be presentable to users in } \\
\text { ways they can perceive. }\end{array}$ \\
2) Operable & $\begin{array}{l}\text { User interface components and navigation must be operable. } \\
\text { 3) Understandable }\end{array}$ \\
$\begin{array}{l}\text { Information and the operation of user interface must be understandable. } \\
\text { 4) Robust }\end{array}$ & $\begin{array}{l}\text { Content must be robust enough that it can be interpreted reliably by a wide } \\
\text { variety of user agents, including assistive technologies. }\end{array}$ \\
\hline
\end{tabular}


Table 2. Principles of Universal Design [Connell et al. 1997].

Principle
1) Equitable use
2) Flexibility in use
3) Simple and intuitive
4) Perceptible informa-
tion

5) Tolerance for error

6) Low physical effort

7) Size and space for approach and use

\section{Description}

The design is useful and marketable to people with diverse abilities.

The design accommodates a wide range of individual preferences and abilities.

The design is easy to understand, regardless of the user's experience, knowledge, language skills, or current concentration level.

The design communicates necessary information effectively to the user, regardless of ambient conditions or the user's sensory abilities.

The design minimizes hazards and the adverse consequences of accidental or unintended actions.

The design can be used efficiently, comfortably, and with a minimum of fatigue.

Appropriate size and space is provided for approach, reach, manipulation, and use regardless of the user's body size, posture, or mobility.

nication technologies (ICTs) for building inclusive, resilient and smart cities and communities; and 5) Full and active participation of persons with disabilities and broad-based multi-stakeholder partnerships for advancing inclusive and accessible urban development.

\section{Case Study: Ubiquitous Interactive Installations in a Museum}
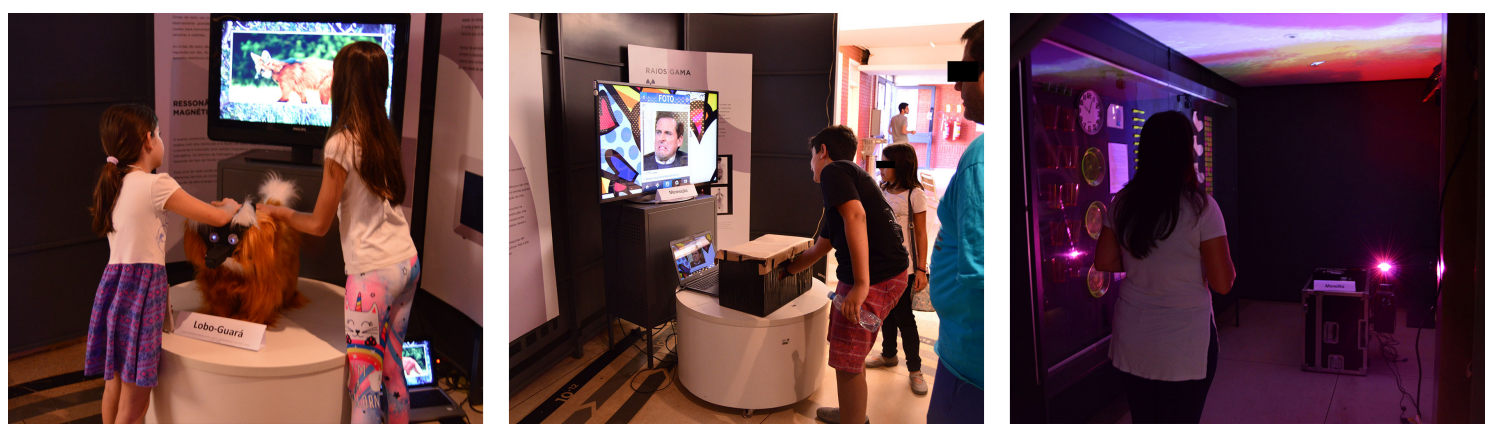

Figure 1. The three artifacts exhibited: Lobo-Guará, Memoção, and Monolito.

Our case study took place on April 21, 2018, at the Exploratory Science Museum of Unicamp, with an workshop entitled "The Magic of Science". A total of $N=15$ children and adolescents participated, some alongside their parents. The activity had an approximate duration of three hours and it was distributed in five phases: (1) reception; (2) exploration of three interactive installations; (3) reflection and discussion about the technologies, (4) construction of a digital artifact, and (5) evaluation of the workshop. Further details of the workshop and its activities are previously published in [Duarte et al. 2019]. This case study analyzed the data from the recorded videos of the workshop. We are therefore interested in the ubiquitous artifacts concerning phase 2: the exploration of the three interactive installations. The three interactive artworks illustrated in Fig. 1 are briefly described as follows:

1) Lobo-Guará is an interactive cardboard maned wolf covered with synthetic fur. It has hidden buttons in its head, body, leg, and tail. When the buttons are pressed they trigger auditory and visual information in a display behind the wolf. For instance, pressing 
the button on the leg provides information about the average size of the wolf's footprints. Furthermore, a proximity sensor in the head detects attempts to pet the wolf, triggering red eyes and a bark sound to communicate that the wolf is a wild and dangerous animal; 2) Memoção is a black box with six textures inside, intended to evoke emotions associated with the textures through Internet memes. Each texture (e.g., rough, soft, gooey) can be touched, and when it happens, a pair of meme and sound related to that texture is played in a display behind the black box. For instance, touching the gooey texture evokes a disgusting meme and sound. To keep the experience non-repetitive, the memes and sound are randomly selected from a curated collection of 10 memes and 2 sounds for each texture inside the box; and 3) Monolito is a miniature monolith inspired by the 2001: A Space Odyssey film, used to interact with a scene from the movie projected with a $360^{\circ}$ projector in a dark room. The audience can pick up the monolith and freely move it around, while an accelerometer and a gyroscope capture the movement to wirelessly cause changes to the projection image. As an example, shaking the monolith can temporarily increase the playback rate and add a red filter to the image.

\subsection{Results}

Good practices of urban environments contributes to equitable access by removing environmental barriers of the ubiquitous environment physical space. The evaluation of the urban space of the museum showed the presence of ramps as a facility to locomo-

Table 3. Applicability of the WCAG 2.1 on the Case Study

\begin{tabular}{|c|c|}
\hline Principle & $\begin{array}{l}\text { Is it suitable to Ubiquitous Environ- } \\
\text { ments? Why? }\end{array}$ \\
\hline $\begin{array}{l}\text { 1) Perceiv- } \\
\text { able }\end{array}$ & $\begin{array}{l}\text { Requires adaptation: Ubiquitous } \\
\text { environments allow open interpre- } \\
\text { tation of the user (e.g Mood Con- } \\
\text { ductor, Monolito). There is no right } \\
\text { or wrong comprehension of the sys- } \\
\text { tem. Culture, experience, beliefs } \\
\text { influence perception and interpreta- } \\
\text { tion. }\end{array}$ \\
\hline
\end{tabular}

2) Operable Requires adaptation: The interface goes beyond the computer screen and keyboard. A big variety of devices allows different types of interaction involving the physical world.

3) Under- Requires adaptation: the operation standable of the ubiquitous environment is not in the focus of attention of the user. The interaction is transparent and Which requirements are missing in the case study?

The addition of multiple outputs such as subtitles, audio description, sign language transcription and audio recordings of the text information would improve the accessibility for deaf, hearing impaired, blind or visually impaired people.

No adaptations are recommended. The artifacts can be touched and manipulated by users with different conditions without apparent issues.

The addition of a short description of what is a Monolito might improve the learning experience of the natural.

4) Robust Not suitable: There is no user agent in ubiquitous environments, the user is already inside of the system. 
Table 4. Applicability of the Principles of Universal Design on the Case Study

\begin{tabular}{lll}
\hline Principle & $\begin{array}{l}\text { Is it suitable to Ubiquitous Environ- } \\
\text { ments? Why? }\end{array}$ & $\begin{array}{l}\text { Which requirements are missing in } \\
\text { the case study? }\end{array}$ \\
\hline
\end{tabular}

1) Equitable Suitable: different possibilities of use interactions at ubiquitous environments require the need of equitable access.

2) Flexibil- Suitable: ubiquitous computing is ity in use able to adapt itself, read and learn the preferences of the user.

3) Simple Suitable: ubiquitous environments and intuitive must not require focus of attention in how to perform the interaction.

4) Percepti- Suitable: ubiquitous computing ble informa- must occupy the periphery of attention tion. The interaction must be natural and transparent.

5) Tolerance Suitable: the prevention of risk sitfor error uations for the user suits the diversity of ubiquitous devices and interaction possibilities.

6) Low Suitable: ubiquitous computing has physical different size and shapes. Not only effort artifacts but also the body of the user becomes inputs on the system.

7) Size and Suitable: environments require difspace for ferent spaces depending on the goal approach of the designed scenario. Little No adaptations are recommended. Exploration is part of the interaction, and the social interactions among participants encourages people to explore the artifacts.

The addition of high contrast option on text information would benefit low vision impaired people.

No adaptations are recommended. The exploration is part of the artifacts' interaction.

Deaf people would benefit from sign language in Lobo-Guará. A coupling of sound and movements in Monolito's animation would improve accessibility for blind people. No adaptations are recommended to the artifacts. There is no right or wrong interaction on this installation. No risky situations are identified.

The possibility of lifting up and down Memoção and Monolito would make the interaction more comfortable to taller people.

No adaptations are recommended. The installation has no barriers to disturb participants' interaction.

and use space would hinder the interaction.

tion. Information about tactile floors, maps or braille instructions were unavailable on the documentation of The Magic of Science. Tables 4 and 3 present the results of the case study. Table 4 synthesizes the applicability of the Universal Design Principles, while Table 3 summarizes and discusses the applicability of the WCAG 2.1 Principles on the case study. Both Tables point out missing aspects in the artifacts regarding accessibility and universal design. Section 3.2 discusses the results on the basis of the research questions.

\subsection{Discussion}

Our findings reveal that the mainstream accessibility principles we found do not cover the entire richness of ubiquitous environments. Furthermore, the dimension of accessibility in ubiquitous environments is not clearly defined yet. The analyses contained in section 3.1 revealed that social, body movements, no interface, free movements, natural interaction, 
exploration and the user being part of the environment are not fully considered.

Column 2 in Tables 4 and 3 illustrates the main findings regarding RQ1 "Are literature guides sufficient to evaluate accessibility in ubiquitous environments?". Although the principles presented are robust, results point out the need for their adaptation to the ubiquitous paradigm of computing systems, including the principles 1- Perceivable, 2Operable and 3 -Understandable from WCAG (and their guidelines as well as success criteria). Further research should adapt these principles to extend their coverage to ubiquitous computing; for example, not having a predefined task to be completed and not interacting only by the click of the mouse or keyboard entrances, but considering the sensors and actuators of ubiquitous environments. Meanwhile, principle 4 - Robust from WCAG is not suitable to ubiquitous environments. Moreover, results indicate as feasible the adoption of Universal Design principles, and of urban good practices, to cover the richness of an ubiquitous environment regarding making it a space for all.

Column 3 in Tables 4 and 3, in turn, illustrates the main findings needed to answer RQ2 "What are the drawbacks to evaluate accessibility in ubiquitous environments?". Some recommendations from the analysis results include multiple output sources, height adjustment for approximation to the installations, and short descriptions for technical words displayed. Also, the adaptation of standards coming from other media (e.g. web) is certainly a huge challenge as the user's own body is a vehicle of interaction in ubiquitous scenarios.

Regarding limitations, this work addressed children and teens between 10 and 15 years old as the targeted audience in the workshop, although some parents and younger siblings also joined the museum exhibition and could also be observed. Among the people who attended the Museum workshop, there were not people with disabilities, preventing us of an indepth analysis of access with those that could be in more need. Furthermore, although the analysis considering only the principles of the formal standards contributes to a preliminary vision of the main issues, extending it to the standard guidelines could enrich the study with technical details of facilitating $x$ preventing access in ubiquitous environments.

\section{Conclusion}

The dynamics of ubiquitous environments brings different possibilities of interactions and new forms of social involvement. The diversity of ways of interacting with the environment, its objects and other people around, typical of ubiquitous computing, raise key-points for considering and developing accessibility evaluation frameworks. The main contributions of this paper are: 1) suitability analysis of existing principles and formal standards for accessibility transposed to the analyzed scenario; 2) From the analysis result, insights on missing aspects that might be brought to the design of ubiquitous computing scenarios, regarding making them accessible for all. Those insights may inform new instruments for designing and evaluating these systems.

We concluded that existing formal standards when placed together contributes to an overview of accessibility in ubiquitous environments. However, they are not enough to cover all ubiquity aspects of the studied scenarios. Social interaction was not covered either. Thus, providing the adaptations and exclusion described on section 3.2 would enlarge the coverage of the accessibility in the studied scenarios. Finally, a deeper analysis 
throughout guidelines and success criteria would enlarge the coverage and point other needs of adaptations.

The study results show that existing formal standards, when placed together, contribute to an overview of accessibility in ubiquitous environments. However, they are not enough to cover all ubiquity aspects of the studied scenarios. Social interaction was not covered for example. Thus, providing the adaptations and exclusion described in section 3.2 would enlarge the coverage of the accessibility in the studied scenarios. Finally, a deeper analysis throughout guidelines and success criteria would enlarge the coverage and point to other needs of adaptations.

New investigations after the release of WCAG 3.0 might bring important results to ubiquitous computing. The development of measurement and evaluation accessibility instruments would support designers towards equitable access. Future works include the evaluation of the socioenactive aspects of ubiquitous systems, regarding the reach of access for all.

\section{Acknowledgments}

This work is financially supported by the São Paulo Research Foundation (FAPESP) through grants \#2015/16528-0, \#2020/04242-2, and by the National Council for Scientific and Technological Development (CNPq) through grant \#304708/2020-8.

\section{References}

Bittencourt, I. I., Baranauskas, M. C., Pereira, R., Dermeval, D., Isotani, S., and Jaques, P. (2015). A systematic review on multi-device inclusive environments. Universal Access in the Information Society, 15(4):737-772.

Connell, B. R., Jones, M., Mace, R., Mueller, J., Mullick, A., Ostroff, E., Sanford, J., Steinfeld, E., Story, M., and Vanderheiden, G. (1997). The principles of universal design.

Duarte, E. F., Maike, V. R. M. L., Mendoza, Y. L. M., de Lima Tenório Brennand, C. V., and Baranauskas, M. C. C. (2019). "the magic of science:" beyond action, a case study on learning through socioenaction. In Anais do XXV Workshop de Informática na Escola (WIE 2019), pages 501-510, Porto Alegre, RS, Brasil. SBC.

Emiliani, P. L. and Stephanidis, C. (2005). Universal access to ambient intelligence environments: Opportunities and challenges for people with disabilities. IBM Systems Journal, 44(3):605-619.

Gonçalves, D. A., Baranauskas, M. C. C., and dos Reis, J. C. (2020). Accessibility in pervasive systems: An exploratory study. In Streitz, N. and Konomi, S., editors, Distributed, Ambient and Pervasive Interactions, pages 25-38, Cham. Springer International Publishing.

Hayashi, E. C. S. and Baranauskas, M. C. C. (2017). Accessibility and affect in technologies for museums. In Proceedings of the XVI Brazilian Symposium on Human Factors in Computing Systems. ACM.

Mendoza, Y. L. M. and Baranauskas, M. C. C. (2019). TangiTime: Design a (Socio)enactive Experience for Deep Time in an Educational Exhibit. In Proceedings of the 18th Brazilian Symposium on Human Factors in Computing Systems. ACM. 
Morgan, E. and Gunes, H. (2013). Human nonverbal behaviour understanding in the wild for new media art. In Salah, A. A., Hung, H., Aran, O., and Gunes, H., editors, Human Behavior Understanding, pages 27-39, Cham. Springer International Publishing.

Stephanidis, C. (2009). Designing for all in ambient intelligence environments: The interplay of user, context, and technology. International Journal of Human-Computer Interaction, 25(5):441-454.

Takayama, L. (2017). The motivations of ubiquitous computing: Revisiting the ideas behind and beyond the prototypes. Personal Ubiquitous Comput., 21(3):557-569.

United Nations (2013). Accessibility and development - mainstreaming disability in the post-2015 development agenda.

United Nations (2015). Good practices of accessible urban development.

W3C (2018). Web content accessibility guidelines 2.1 (wcag).

Weiser, M. (1991). The computer for the 21st century. Scientific American, pages 94-104.

World Health Organization (2011). World report on disability 2011.

World Health Organization (2013). How to use the icf: A practical manual for using the international classification of functioning, disability and health (icf) : Exposure draft for comment. 